\title{
The Effect of Moral Competence on Online Conformity Behavior
}

\author{
Aida Mofakhami \\ (Allameh Tabataba'I University, Teheran; Iran; aida.mofakhami@gmail.com) \\ ORCID: 0000-0002-3390-6488
}

\begin{abstract}
Moral Competence, defined as the ability to solve conflicts on the basis of shared moral principles through cooperation rather than through violence, deceit and power, has received little attention among different psychological approaches; despite its importance in predicting many of our social interactions. The purpose of this study was to investigate the effect of moral competence on online conformity behavior. 217 students from universities in Teheran were selected for a quasi-experimental study. First, participants' moral competence was measured with the online Moral Competence Test (MCT) by G. Lind (1978/2019). Then the subjects participated in an online version of an Asch type experiment in which conformity was induced. The results showed a clear conformity behavior in the use of the internet. An average of $32.09 \%$ of participants conformed to each critical question. When compared to Asch`s line judgment task, the mean conformity in this experiment was lower, but still significant enough to indicate conformity behavior (36.8\% compared with 7.4\%), which might stem from the online situation, in which some other variables like the deindividuation effect might influence this difference. The results also indicated that there was a weak but negative correlation between moral competence and conformity behavior. The results confirm our hypothesis weakly; subjects with higher moral competence tended to show lower conformity. If the results could be replicated, it would imply that conformity is not a general and stable trait of people, as Asch assumed, but depends on people's level of moral competence, which can be fostered through education.
\end{abstract}

Keywords: morality; moral competence; social conformity; online conformity; MCT; experimental research with university students.

\section{Introduction}

Conformity as a psychological term is defined as an influence resulting from one's willingness to accept others' opinions about reality (Asch 1955). Although it is human nature to follow others, conformity can lead to very dangerous behaviors. As Zimbardo (2007) has noted, conformity is a strong group psychological mechanism that can make 
people behave inhumanly. On the other hand, conformity as a social mechanism can also serve as a prosocial behavior, helping group dynamics and more effective communication (Bond \& Smith 1996). The examples of this behavior occur in almost all our everyday life contexts, e.g., when we stand while our national anthem is played as others do so, or when we "like" others' posts on social media such as Instagram just because they have been liked by a great number of other people; and generally, when we confirm to ideas just because the majority has accepted them, not because we truly believe in them. In such situations we are simply conforming. As the internet has penetrated every facet of our lives and has become inseparable from day-to-day conduct, it is necessary to take into account this emerging context when studying such variables in the social interactions of individuals. With this in mind, the current study was designed to take an online form, in order to investigate online conformity behavior.

Solomon Asch (1958) was one of the first psychologists to study conformity. He used a lab experiment in which a group of eight persons participated in a "line judgment" task, when in fact only one of them was the real participant, and the other seven were confederates/stooges who acted as participants, while the real participant was unaware of this. During the experiment, each student viewed a card showing a target line on one side and three comparison lines on the other side. Each person in the room had to choose aloud which comparison line (A, B or C) was similar to the target line. The answer was always obvious. The real participant was always the last person who had to give their answer, and the confederates had agreed in advance to give an obviously wrong answer in most trials (critical trials). On average, about one third of the participants who were placed in this situation sided with the clearly wrong majority in the critical trials. Asch's experiment $(1955,1956,1958)$ also featured a control condition where there were no confederates, only "real participants". In Asch's line judgment task, the test subjects complied on average with the majority's wrong judgment in $36 \%$ of their selections (Rosander \& Eriksson 2012). Although conformity as a face-to-face behavior has been widely studied by many previous researchers (Deutsch \& Gerard 1955; Bond \& Smith 1996; Baumeister 1982; Janes \& Olson 2000; Goeree \& Yarive 2015), to this day there are not many studies that have investigated conformity behavior in non face-to-face situations.

With regard to recent studies on conformity in CMC (computer mediated communication) conditions, Rosander and Eriksson (2012) authored Conformity on the Internet and The Role of Task Difficulty and Gender Differences. These are well-designed studies, from which the present study draws inspiration. Rosander and Eriksson used a web-based survey as the social context for their study, and the results showed that $52.6 \%$ conformed at least once, with an average $13.0 \%$ of participants conforming on each critical question. The conformity increased with higher task difficulty, and no difference was found between men and women in terms of their conformity behavior. The study discussed some reasons for this form of conformity behavior, based on theories and previous studies, 
such as turning to the group for guidance, avoiding social isolation and protecting one's self-esteem (Rosander \& Eriksson 2012). However, Rosander and Eriksson placed more emphasis on environmental factors that influenced conformity, such as social isolation. The importance of more internal variables, which seem to differ due to education, have received little attention so far, yet they may also play an essential role in conformity behavior. In other words, conformity may not be as static as Asch assumed.

In the present study, Moral Competence comes into play as another variable. We assume it has animpact on conformity behavior, on the basis of its theoretical underpinnings and its definition. This concept was first introduced by Georg Lind (1978), the German psychologist and philosopher. He defines moral competence "as the ability to solve conflicts and problems on the basis of shared moral principles through thinking and discussion rather than through violence, deceit and power." Specifically, it is the ability to rate the arguments of others with regard to their moral quality rather their opinion-agreement. This study considers moral competence as a key variable which plays an essential role in the extent of an individual's conformity behavior.

Conformity may also play a role when people behave immorally when others ask them to do so, even when their own moral principles would not allow this. Stanley Milgram (1963) has shown that two thirds of his participants gave other subjects electroshocks in an alleged learning experiment, even though they thought this was wrong, as they said afterwards. Lawrence Kohlberg (1984) repeated Milgram's experiment, showing that participants with high moral competence refused to obey this immoral instruction. They seem to be immune to conforming with immoral orders. Perhaps the influence of moral competence on conforming behavior can also be demonstrated with the current experiment.

\section{Main Hypotheses}

As was mentioned above, there is evidence for conformity behavior in CMC, but the strength of such behavior is considerably lower when compared with face-to-facesituations, as investigated by Asch (Rosander \& Errikson 2012; Bond \& Smith 1996). Therefore, we replicated this method in order to investigate whether the previous results are consistent in different cultural settings. For this reason, only Iranian students participated in this experiment.

On the other hand, as the definition of moral competence suggests, the more people were able to judge arguments on the basis of their own principles (and not under the influence of other opinions), the less they will conform to the wrong majority options. In other words, people with higher moral competence are less likely to express conformity behavior in conditions characterized by social pressure. Therefore, we propose:

(1) Conformity behavior would occur in an online context;

(2) People with high moral competence are less likely to succumb to the pressure to conform. 


\section{Research Methodology}

\subsection{Participants}

There was a total of 217 participants, consisting of 140 women and 77 men $71.7 \%$ females and $28.3 \%$ males), ranging from 18 to 36 years old, and they all participated voluntarily. They mostly came from the website to which this experimental design was uploaded (www.Ravanhami.ir) and received a cash credit as a reward for their participation at the end of the experiment. Furthermore, regarding ethical considerations, all the participants received an email explaining the true aim of the research after the deadline for the survey.

The population of this study compromised undergraduate students of three universities in Teheran in the Winter Semester of 2017-2018.

The subjects were divided into two separate groups (one control group and one conformity group). 10 participants were eliminated from the sample because they were under 18 or did not complete the whole experiment.

\subsection{Materials}

\subsubsection{MCT (Online form)}

The Moral Competence Test (MCT) contains 24 arguments pro and contra the protagonists' decision in two dilemma situations which are to be rated with regard to their acceptability. They are first asked to express their opinion about the actor's decision (the first six arguments are in support of the actor's decision and the other 6 arguments contradict his decision) and then, regardless of their opinion, they are asked to rank the arguments on a Likert scale ranging from -4 (strongly disagree) to +4 (strongly agree). Each argument was designed to represent one of Kohlberg's six type of moral orientation (Lind2019). Depending on the pattern of their answers, the respondents' moral competence is scored and receives a score ranging from 0 (no moral competence at all) to 100 (very high moral competence). The MCT is suitable for people over 10 years old.

The implicit task of the MCT is to rate the arguments with regard to their moral quality instead of in terms of whether participants agree with the arguments. That is, this task was not made explicit to the subjects. In this study, the Persian translation of the MCT by Saeidi (2011) was used in an online form, which was available on a web site named www.ravanhami.ir. 
Doctor

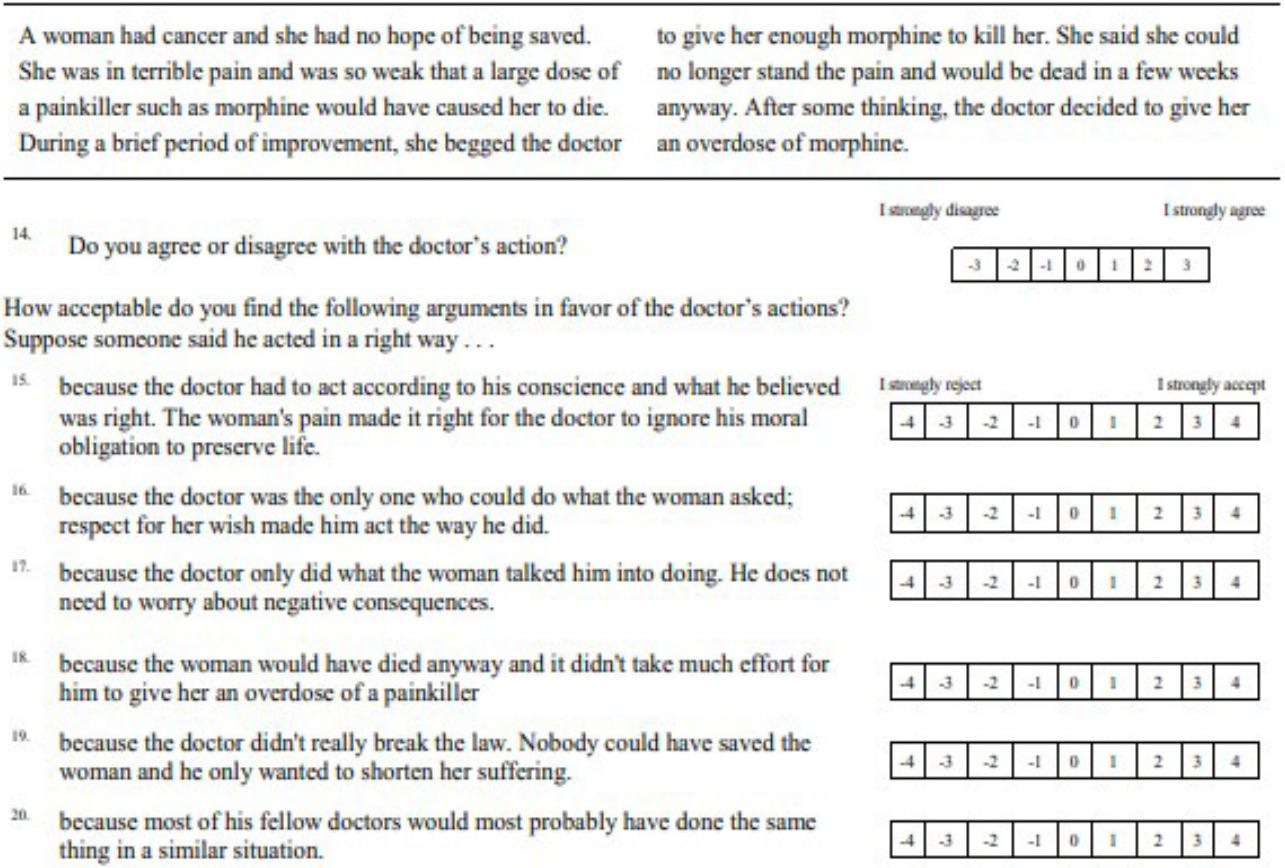

Figure 1: MCT by G. Lind (1978/2019). Doctor-Dilemma with six supporting arguments as an example.

\subsubsection{Conformity situation (Web-based survey)}

In order to induce conformity behavior, the author designed an experimental situation which was methodologically a replication of Rosander and Eriksson (2012) but customized the question in a way that matches to the participants' cultural background. In this situation, the participants were randomly assigned to one of the two groups, in which they were asked to answer 40 questions about general knowledge in four areas including, chemistry, history, literature and geography. Participants in the experimental group were presented with a fake diagram showing that the great majority of the previous participants had chosen an obviously wrong option, while the control group received no diagrams for the same questions (Figure 2). Conforming answers were given the value of one and nonconforming answers were given the value of zero.

Question 12. What is the chemical symbol for calcium?

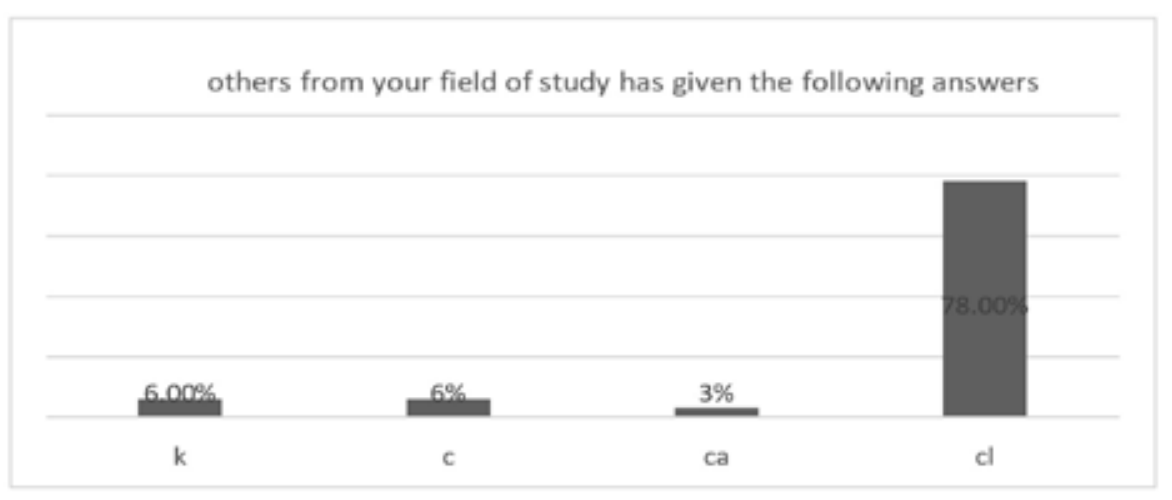

Figure 2: an example question shown to THE experimental group (critical question). 

a. $\quad \mathrm{k}$
b. C (conformity answer)
c. Ca (correct answer)
d. $\quad \mathrm{Cl}$

\subsection{Research design and procedure}

The study was quasi-experimental, with an experimental condition. In fact, the fake diagrams played the role of confederates in Asch's classic experiment, because they showed that the majority of participants had selected the obviously wrong answer.

In about $40 \%$ of the cases, the correct description of the answer's distribution was displayed on diagrams called "neutral question" and the distribution in the other $60 \%$ of the diagrams was fabricated, showing the majority chose an incorrect answer, called "critical questions" (Rosander \& Eriksson 2012). The reason for having 40\% of neutral question was to avoid raising too much suspicion in the participants, since a high level of suspicion seems to have a negative influence on conformity behavior and results in methodological problems (Stang 1976).

The procedure of this study included 3 steps:

Step 1: All the participants completed one page of demographic questions (age, gender and education level).

Step 2: They answered the online form of MCT questions before starting the experimental phase.

Step 3: They were assigned to one of the two groups and were asked to answer 40 items in the general knowledge test. The order of questions was identical in both the control and experimental groups.

In order to prevent the web search effect for the experimental part of the research, the participants had only 30 seconds to answer each question and they could only go forward during the experiment. They were not allowed to use the "back" option during the test.

\section{Research Results}

For the statistical analysis of the two main hypotheses of this study, PSPP were used. No significant differences were found regarding the age, gender differences and educational levels between the conformity and control groups.

\subsection{Conformity behavior would occur in an online context}

With regard to this assumption, we expected a significant difference in conformity levels between the control and experimental groups. For the twenty-two critical questions, the mean number of answers in accordance with the manipulation in the conformity group $(M=7.482, S D=4.421, N=112)$ was higher than the control group $(M=3.284, S D=1.766$, 
$n=96)$. As can be seen, the difference between the mean scores of the experimental and control groups suggests that in this experimental situation conformity does occur; that is, even people who are alone when using the internet still succumb to the pressure of the majority and conform (Figure 3).

In the second analysis, conformity was measured as difference: for each of the 22 critical questions, there was a difference between the number of answers matching the incorrect and fabricated majority answers showed to the conformity group and the number of participants giving the same answers in the control group. $\chi^{2}$ was used to determine if the frequency of answers matching the manipulation in the conformity group differed from the frequency of the same answers in the control group.

According to the results of the $\chi^{2}$ test, there was a significant difference between the experimental and control group for all the questions, except three of them (q15, q23 and q30). So the experimental group conformed much more than the control group.

Both analyses (mean scores and $\chi^{2}$ test) support hypothesis 1: the conformity group conformed to what they were led to believe was the answer of the majority of the participants to a greater degree than the chance of participants in the control group giving the same incorrect answer. The results show that conformity behavior occurs in an internet-based context. Although it is not as great as what Asch observed, it is still remarkable.

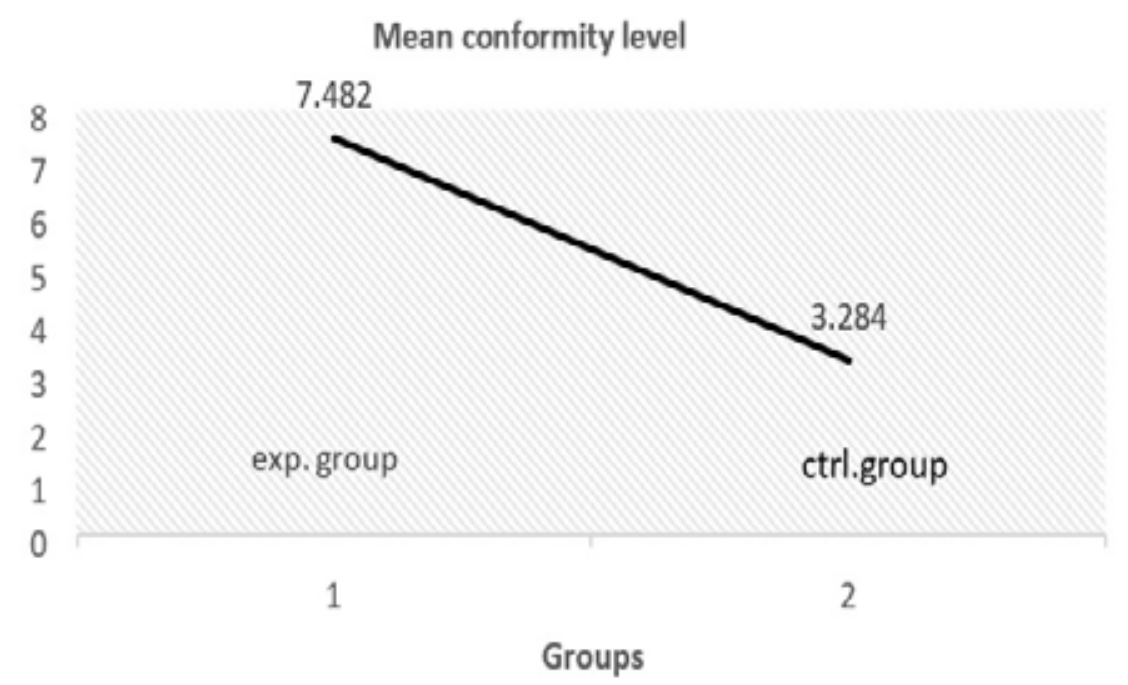

Figure 3: Comparing mean conformity levels between experimental and control groups.

\subsection{People with high moral competence are less likely to succumb to conformity pressure}

According to this hypothesis, we expected a significantly lower level of conformity behavior among the high C-score group.

The mean score for moral competence in the experimental group ( $M=20.552$, 
$\mathrm{SD}=16.379, \mathrm{~N}=112)$ was almost compatible with the control group $(\mathrm{M}=20.821, \mathrm{SD}=15.71$, $B=96$ ). The results reveal that participants with moral competence with $C$ (for moral competence) higher than 20 ( $C>20)$ show less conformity than participants with lower than 20 ( $C<20)$. Moral competence and conformity behavior were negatively correlated $(r=-0.183)$. Higher C-scores are compatible with a decrease in conformity behavior in participants (Figure 4). As can be seen in Figure 4, the correlation is rather small. This occurs maybe for the following reason: the pressure to conform was not high enough to show a wider difference in the conformity level for different C-scores, but as can be seen in Figure 2, these two variables are still negatively correlated.

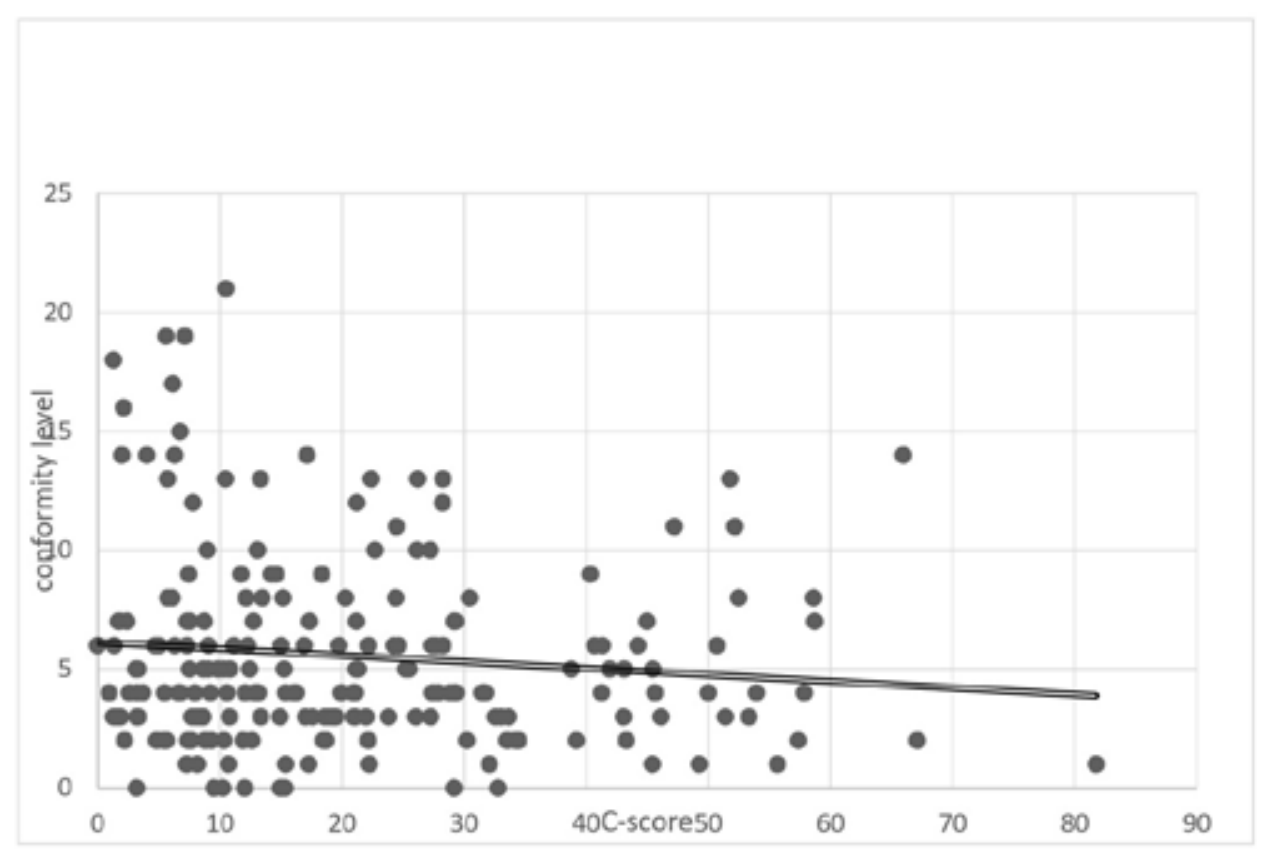

Figure 4: Participants' conformity level with different C-scores.

\section{Discussion}

The main purpose of this study was to investigate the impact of moral competence on conformity behavior in a non-face-to-face situation. As the results indicate, there is a weak but negative correlation between moral competence score and conformity behavior. When compared to Asch's results, there seems to be a great decrease in conformity behavior as presented by the participants. We assume the effects of deindividuation may be one of the main reasons for this decrease, as Rosander and Eriksson (2012) also reported. But the evident decline in mean conformity level is consistent with other previous studies of online conformity (Rosander \& Eriksson 2012; Jim \& Park 2011; Cinnirella \& Greene 2007). Comparing to Rosander and Eriksson (2012), the mean conformity of this study is also lower (7.4\% compared with $13.0 \%$ ), which may relate to the differences in culture, experimental design and the sample size. As there are few studies in this area, further replications of this study are needed with a larger sample and with some minor changes in the experimental design, in a manner that will increase the amount of social pressure. 
Some suggestions in this regard:

- The questions which were chosen for the experimental group could be selected more accurately in terms of difficulty, so that the pressure to conform increases; thus a standard pilot study may be needed prior to the experiment.

- Any measure that decreases the deindividuation effect would boost the conformity effect; for example, if participants were told that their answers would be displayed publicly on the website after the test, with their own e-mail address visible, it may influence their behavior to some extent.

In accordance with Hypothesis 2, the results show that moral competence can play a role in conformity behavior. In other words, conformity behavior may not be as fixed as Asch suggested. Rather, moral competence seems to be a mediator variable which affects the level of conformity. As the present research was a preliminary study in this area, a more accurate experimental design with a larger sample size is needed to address this subject in a more reliable manner.

On the other hand, in his conformity experiments Solomon Asch showed that more often than not people value others' opinions more than their own, even when the answers provided by the majority are obviously wrong. Explanations for this behavior, such as participants turning to the group for guidance and avoiding social isolation, have been discussed in earlier research, but all of these factors can be considered environmental, that is, a changing social environment seems to be the only factor that may affect the level of conformity behavior.

However, in this study an "internal variable" has been found which seem to be stronger than other previous ones. Moral competence is an ability which facilitates individuals to act on the basis of their own opinions, regardless of how intense the pressure is on the outside. As we could see in the results, people with higher C-scores were less likely to accept the others' answers, even when the pressure to conform was high due to the fabricated majority answers. In contrast, people with low or medium C-scores conformed to almost every critical question. In addition, there were few individuals in Asch's experiments who never conformed during the test trials, which may be another hint for researchers to focus more on internal reasons rather than environmental ones. Perhaps there is a need for a new model of behavior, an internal model which includes such characteristics as moral competence interacting with conformity behavior. One of the main goals of this study was to find a new way of understanding the influence of social media on people's behavior, since they have recently become a major issue in Iranian society. As Lind (2016) states, moral competence is an educational concept which can be learned through development. According to his definition, conformity behavior seems to change through learning moral competence. This could be one of the new main policies of educational systems all around the world, especially in developing countries like Iran. 


\section{Conclusions}

The present study aimed to see if and how people conform in an online setting and how moral competence would affect people's conformity behavior in this context. The results show that people do conform in this situation; that is, even people who are online by themselves are still under the pressure of the majority to produce conformity behavior. This is consistent with earlier research (Rosander \& Eriksson 2012; Jim \& Park 2011; Cinnirella \& Greene 2007). When compared to Asch`s line judgment task, the mean conformity in this experiment is lower (36.8\% compared with $7.4 \%$ ), which is because of many differences in experimental design, especially the online form of experiment which is significantly different from face-to-face situation.

Overall, although this result was rather weak in comparison with other face-to-face experiments, it still could indicate that people's level of moral competence could affect their social behavior, even in an online platform. In other words, people with higher moral competence, which can be acquired through education, are less prone to showing conformity in conditions characterized by social pressure.

\section{References}

Asch S. E. 1955. “Opinions and Social Pressure," Scientific American 193(5):31-35.

Asch S. E. 1956. "Studies of Independence and Conformity. A Minority of One Against a Unanimous Majority," Psychological Monographs 70(9) (whole No. 416):1-70.

Asch S. E. 1958. "Effects of Group Pressure upon the Modification and Distortion of Judgements," in E. E. Maccoby, T. M. Newcomb, \& E. L. Hartley (Eds.), Readings in Social Psychology (pp. 174-183) New York: Holt, Rinehart, and Winston.

Baumeister R. F. 1982. “A Self-presentational View of Social Phenomena,” Psychological Bulletin 91(1):3-26.

Bond R. \& Smith P. B. 1996. "Culture and Conformity: A Meta-analysis of Studies Using Asch's Line Judgment Task," Psychological Bulletin 119:111-137.

Chen Y., Harper F. M., Konstan J., \& Li S. X. 2010. „Social Comparisons and Contributions to Online Communities: A Field Experiment on Movie Lens," American Economic Review 100:1358-1398.

Cinnirella M. \& Green B. 2007. “Does 'Cyber-conformity' Vary Cross-culturally? Exploring the Effect of Culture and Communication Medium on Social Conformity," Computers in Human Behavior 23:2011-2025.

Deutsch M. \& Gerard H. 1955. "A Study of Normative and Informational Social Influences upon Individual Judgement," Journal of Abnormal and Social Psychology 51:629636. 
Goeree J. K. \& Yariv L. 2015. “Conformity in the Lab," Journal of the Economic Science Association 1(1):15-28.

Janes L. M. \& Olson J. M. 2000. “Jeer Pressure: The Behavioral Effects of Observing Ridicule of Others," Personality and Social Psychology Bulletin 26(4):474-485.

Kim J. \& Park H. S. 2011. „The Effect of Uniform Virtual Appearance on Conformity Intention: Social Identity Model of Deindividuation Effects and Optimal Distinctiveness Theory," Computers in Human Behavior 27:1223-1230.

Kohlberg L. \& Candee D. 1984. "The Relationship of Moral Judgment to Moral Action," in W. M. Kurtines \& J. L. Gewirtz (Eds.), Morality, Moral Behavior, and Moral Development (pp. 52-73). New York, NY: Wiley.

Lind G. 1978. „Wie misst man moralisches Urteil? Probleme und alternative Möglichkeiten der Messung eines komplexen Konstrukts“ [How does one measure moral judgment? Problems and alternative ways of measuring a complex construct], in G. Portele (Ed.), Sozialisation und Moral (pp. 171-201). Weinheim: Beltz.

Lind G. 1989. "Measuring Moral Judgment: A Review of 'The Measurement of Moral Judgment' by Anne Colby and Lawrence Kohlberg," Human Development 32(6):388-397.

Lind G. 2000 (Orig. 1984). Content and Structure of Moral Judgment ( $2{ }^{\text {nd }}$ Corrected Edition). Doctoral Dissertation, University of Konstanz.

Lind G. 2016. How to Teach Morality: Promoting Deliberation and Discussion Reducing Violence and Deceit. Berlin: Logos Verlag GmbH.

Milgram S. 1963. "Behavioral Study of Obedience," The Journal of Abnormal and Social Psychology 67(4):371-378.

Rosander M. \& Eriksson 0. 2012. "Conformity on the Internet - The Role of Task Difficulty and Gender Differences," Computers in Human Behavior 28(5):15871595.

Saeidi-Parvaneh S. 2011. Moral, Bildung und Religion im Iran: Zur Bedeutung universitärer Bildung für die Entwicklung moralischer Urteils-und Diskursfähigkeit in einem religiös geprägten Land (Doctoral Dissertation, University of Konstanz).

Stang D. J. 1976. "Ineffective Deception in Conformity Research: Some Causes and Consequences," European Journal of Social Psychology 6:353-367.

Zimbardo P. 2007. The Lucifer Effect: Understanding How Good People Turn Evil. New York: Random House. 